\title{
SOBRE HOMENS, CÂNCER DE PRÓSTATA E SAÚDE: UMEN- SAIO A LUZ DA ANTROPOLOGIA DAS MASCULINIDADES
}

\section{Jeferson Santos Araújo' \\ Márcia Maria Fontão Zago²}

Resumo: Este estudo oferece uma visão sobre a influência das masculinidades frente ao ajustamento dos homens à doença e aos tratamentos para o câncer de próstata (CP). Trata-se da síntese de uma tese de doutorado a qual foi desenvolvida pela perspectiva qualitativa, adotando como método a etnografia narrativa e o referencial teórico da Antropologia Médica. Participaram do estudo 17 homens diagnosticados em seguimento terapêutico em um hospital universitário localizado no interior do estado de São Paulo. Duas unidades temáticas ilustram os significados das experiências investigadas: enredos corporificados do câncer de próstata: normas, conflitos e descobertas; e masculinidades resignadas em tempos de crise: como nos vemos, enfrentamos e seguimos no nosso mundo social. Foi possivel identificar que a cultura influenciou na experiência do homem adoecido pelo CP, transformando seu corpo biológico, social e sua masculinidade.

Palavras-chave: antropologia médica; neoplasias da próstata; masculinidade.

Abstract: This study provides insight into the influence of masculinities on men's adjustment to disease and treatments for prostate cancer (PC). It is the synthesis of a doctoral thesis in which it was developed by the qualitative perspective, adopting as method the narrative ethnography and the theoretical reference of Medical Anthropology. Participants were 17 men diagnosed in a therapeutic follow-up at a university hospital located in the interior of the state of São Paulo. Two thematic units illustrate the meanings of the experiences investigated: embodiments of prostate cancer: norms, conflicts and discoveries; and resigned masculinities in times of crisis: as we see ourselves, we face and follow in our social world. It was possible to identify that the culture influenced in the experience of the man sick by the PC, transforming its biological body, social and its masculinity.

\footnotetext{
Doutor em Enfermagem pela Universidade de São Paulo. Docente da Universidade Federal do Sul e Sudeste do Pará. E-mail. araujojs@unifesspa.edu.br

2 Doutora em Enfermagem pela Escola de Enfermagem de Ribeirão Preto da Universidade de São Paulo Professora associada a Escola de Enfermagem de Ribeirão Preto da Universidade de São Paulo.
} 
Keywords: medical anthropology; prostate neoplasms; masculinity.

\section{Introdução}

câncer de próstata (CP) é a neoplasia maligna mais incidente entre os homens brasileiros, depois do de pele não melanoma. No nosso país, a idade média na qual o CP é mais comumente diagnosticado é entre 72-74 anos. Os procedimentos diagnósticos incluem o exame digital transretal da próstata, avaliação do antígeno prostático específico (PSA) e a biopsia por ultrassonografia transretal. Entretanto, os adenocarcinomas de próstata têm evolução longa até tornarem-se sintomáticos. Por outro lado, o risco de um toque prostático positivo representar um câncer é dependente do valor do PSA, e os outros procedimentos são necessários para a confirmação do diagnóstico (RHODEN e AVERBECK, 2010; BRASIL, 2011; NATIONAL INSTITUTES OF HEALTH/NATIONAL CANCER INSTITUTE, 2012).

Os homens com CP lidam com dificuldades devido ao diagnóstico do câncer e aos efeitos dos tratamentos que alteram a sua qualidade de vida. Estudos evidenciam que eles podem desenvolver incontinência urinária e impotência sexual após os tratamentos. Após cinco anos de tratamento, a disfunção sexual erétil pode ocorrer em $80 \%$ dos pacientes submetidos à prostatectomia radical e em $50 \%$ dos submetidos apenas à radioterapia externa (MIGOWSKI e SILVA, 2010).

Ezer e colaboradores (2012) investigaram o senso de coerência, coesão e adaptação com a parceira, sintomas urinários e sexuais e distúrbio do humor, como indicadores objetivos do ajustamento psicossocial de homens com CP, entre 211 canadenses, no primeiro ano após o diagnóstico, em três momentos distintos, por diferentes instrumentos de avaliação. Conclúram que o primeiro ano após o diagnóstico é um período complexo para os adoecidos, que mostraram deterioração nas relações sexuais, familiares e sociais, devido à presença dos indicadores citados.

Entre os vários estudos disponiveis na literatura, o foco na sexualidade do adoecido por CP, por meio de abordagens metodológicas qualitativas, prevalece. Pesquisadores brasileiros investigaram as experiências de vida de dez brasileiros do sudeste do país com incontinência urinária e disfunção erétil após prostatectomia radical, na abordagem clínico-interpretativa. Por meio 
de três temas - alterações na sexualidade, corpo sem governo e perdas - os participantes relataram sobre as dificuldades em lidar com a impotência física e emocional resultante do tratamento, com sentimentos de baixa autoestima e angústia psicológica (LOPES et al., 2012).

Os latinos e afro-americanos foram os pacientes do estudo de Maliski et al. (2008) sobre a identidade masculina após o tratamento para o CP. Os 91 informantes destacaram as alterações nas funções sociais devido à incontinência e disfunção sexual. No processo de renegociação da identidade masculina, eles empregaram a estratégia da normalização, esperança na melhora e redefinição das prioridades da vida.

Um estudo etnográfico com abordagem na masculinidade, como sinônimo de sexualidade, foi realizado por Cecil, McCaughan e Parahho (2010), com oito irlandeses com cânceres em estágio avançado, que não estavam sendo ativamente tratados. Para estes homens, a doença provocou preocupações econômicas, alterou significativamente seus relacionamentos com a família e amigos, e com a autoimagem.

Os estudos apresentados apontam que, independente do forte avanço nos métodos diagnósticos, o CP ocupa uma fiel posição na vida do homem, sendo os sinais, os sintomas e os tratamentos característicos da doença, responsáveis por proporcionar ao adoecido momentos de sofrimento, medo e incerteza pelo seu prognóstico (KAZER et al., 2011).

Neste sentido, pesquisadores como Chapple e Ziebland (2002), Wall e Kristjanson (2004), Oliffe e Thorne (2007), Maliski et al. (2008) e Cecil, McCaughan e Parahoo (2010)discutem a importância de estudos sobre a experiência do adoecido pelo $C P$, numa perspectiva ampla que relaciona o homem, sua sexualidade, sua cultura e seus significados, com base no conceito de masculinidade, na abordagem da antropologia das masculinidades, e pela metodologia qualitativa.

Os aspectos que influenciam o ajustamento dos homens à doença e ao tratamento são seus conhecimentos, normas e valores culturais, ou seja, as maneiras de pensar, sentir e agir baseadas nas normas implícitas do seu meio 
social, especialmente aquelas que fazem juizo aos roteiros tradicionais de masculidade como ser forte, viril, insensivel e sexualmente potente (BURNS e MAHALIK, 2007; DÁZIO, SONOBE e ZAGO, 2009). O adoecido por $\mathrm{CP}$ é um forte candidato a privar-se desses atributos durante o tratamento, necessitando de apoio social e emocional contínuos.

Masculinidade é uma construção sociocultural relacionada a um tempo histórico específico, à cultura e ao ambiente. Por exemplo, na cultura ocidental, a masculinidade dominante ou hegemônica contemporânea está associada a ser da "raça branca", heterossexual e pertencer à classe média. O estereótipo masculino é de um ser assertivo, com poder, força física e controle emocional (CONNELL, 2005; CONNELL e MESSERSCHMIDT; 2005; EVANS et al., 2011). Porém, nem todos os homens assumem esse modelo, podendo haver diferentes expressões do masculino num mesmo contexto social, como as masculinidades de cumplicidade, de resistência, de subordinação e a marginalizada. As discussões do que é ser homem e do gênero masculino não indicam apenas os comportamentos interpessoais a serem adotados e esperados socialmente, mas, também influenciam no comportamento em saúde e na forma como os homens lidam com seus corpos após o CP (CONNELL, 2005; CONNELL e MESSERSCHMIDT; 2005; EVANS et al., 2011).

\section{Em direção a pesquisa de campo}

Frente a estas colocações e envolvidos pelo desejo de conhecer como é a experiência do homem com o CP e os seus tratamentos e como ele lida com sua masculinidade, foi realizada uma pesquisa qualitativa, adotando como método a etnografia narrativa (CRANG e COOK, 2007) e o referencial teórico da Antropologia Médica (LANGDON, 2003; KLEINMAN, 1988) e das Masculinidades (CONNELL, 2005).

Para melhor compreender os processos de adoecimento dos homens, há a necessidade de se reconhecer a importância da vida cotidiana e dos sentimentos humanos, por metodologias que privilegiem a dimensão subjetiva da masculinidade. É pela subjetividade que analisamos os sentidos específicos da experiência com o adoecimento, sem deixar de relacionar o singular com a perspectiva social mais 
ampla (CONNEL, 2005; POPE; MAYS, 2009).

Como o foco deste estudo foram as subjetividades da experiência do homem com o CP com atenção às masculinidades, a metodologia qualitativa ou interpretativa é a que possibilitou obter informações sobre as sutilezas e complexidades das respostas humanas atribuídas às suas experiências sobre a doença e aos seus tratamentos, essenciais para a construção de intervenções de enfermagem culturalmente sensiveis e efetivas (POPE e MAYS, 2009).

Traçar uma interpretação antropológica para a masculinidade é compreender que de alguma maneira a cultura influencia na forma de pensar e agir do homem frente ao seu corpo, na definição de sua identidade, gênero e sexualidade. Segundo Courtenay (2010), é este modo de pensar e agir que se relaciona diretamente com a maneira como os sujeitos observam e cuidam dos seus corpos, na manutenção de suas crenças e comportamentos de saúde.

Wenger (2013) compreende que quando os homens e mulheres estão inseridos em uma cultura, eles aprendem vários significados para a sua forma física e recebem diretivas sociais relacionadas com o funcionamento físico e emocional através de seus corpos, e ao apresentarem esses corpos para a sociedade, indicam sua concordância ou divergência com os padrões de gênero locais. Wenger (2013) afirma que é no cruzamento dinâmico entre físico e o social que o gênero não pode ser analisado separadamente das questões de saúde e doença, pois é aí que está incluso a forma como os homens e mulheres percebem e entendem suas experiências frente à doença.

A presente pesquisa foi defendida pelo pesquisador Jeferson Santos Araújo sob orientação da Dra Márcia Maria Fontão Zago, na Escola de Enfermagem de Ribeirão Preto da Universidade de São Paulo - USP no dia 22 de julho de 2016, com o titulo "A experiência do homem com câncer de próstata na perspectiva da antropologia das masculinidades". Participaram do estudo 17 homens diagnosticados com CP em seguimento terapêutico em um hospital universitário localizado no interior do estado de São Paulo. A coleta de dados foi realizada de forma individualizada com cada participante por meio de entrevistas em profundidade e observação do contexto de pesquisa, sendo estas registadas em diário de campo, realizada nas dependências do complexo hospitalar e nos lugares 
em que os participantes julgavam propícios para conversar sobre sua experiência.

A captação dos dados ocorreu entre o período de março de 2013 a março de 2016.

Em seguida, os depoimentos foram submetidos a análise temática indutiva e os aspectos semelhantes e particulares das narrativas foram integrados permitindo que obtivéssemos um panorama geral do modelo explanatório dos sentidos compartilhados nas experiências dos homens adoecidos (Quadro 1).

\begin{tabular}{|c|c|c|}
\hline Antes do tratamento do CP & Durante o tratamento do CP & Depois do tratamento do $\mathrm{CP}$ \\
\hline quáo da próbieta & - Diagnestico (mapũo) & - Mudança no fluce de vida por comta das \\
\hline$v e m d o c P$ & - Escollha do tretamemo & complicepêtes após a trafamemsto do CP \\
\hline Destino & $\checkmark$ Radioferapia; quimibterapla; cirngrgia; & $\checkmark$ Incontinència \\
\hline Herance & hormonioteropia & iner cam a urina soltar \\
\hline Castigo par algo que fez & $\checkmark$ Fotares que infuenciaraw & Uso de froilda \\
\hline is e sintamas & r Viver sem sero & - Uso de absanente \\
\hline Vher cow a wrina presa & - Familia & iso de mecia-peniana \\
\hline mes de dereçao $X$ Masculinidade & $>$ Amigas & $\checkmark$ Diminnuiçùo da libido sexnel \\
\hline $\begin{array}{l}\text { Reacdo do madico ao hocar owtro } \\
\text { homem - perspectiva do porieme }\end{array}$ & $\begin{array}{l}\text { Incapocidade de nsar autros } \\
\text { mérodbs }\end{array}$ & $\begin{array}{c}\text { A importaincia da vida semal } \\
\text { - Sero por obvigapabo }\end{array}$ \\
\hline O tahu de faser exame cam mithica & $\checkmark$ Corporifficegpab da preistata retirada & $\checkmark$ Disfingsùo eníif \\
\hline mulher & cirurgicasemte & $>$ Uso de viagra \\
\hline PSA & & Viso de prétese \\
\hline Toque & & - Uso de outros midodas iseco ardi, \\
\hline Biopsia & & masturbaçalo, liga de dimbeina.y \\
\hline Comparaciso entre os erames & & $\checkmark$ Gasar seco (anséncia de giaculagsio) \\
\hline Influsimcia dos dialetas que cercom a & & Andropansa \\
\hline mascwlinidade do homew que se & & Mudanga na alimentogido \\
\hline sudmetent as crames de detecpâto & & $\checkmark$ Muclanca no tratalho \\
\hline do $C P$ & & \\
\hline cwlimidode & -Masculinidade & Mesculinidade \\
\hline 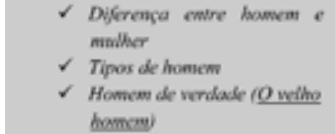 & $\begin{array}{l}\checkmark \text { Mudanga de paradigmas fremte à cirargia } \\
\checkmark \text { Medo, incertesa e sofrimento }\end{array}$ & 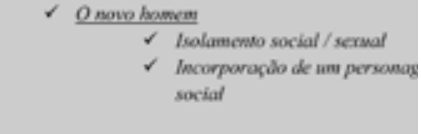 \\
\hline
\end{tabular}

A análise temática trata-se de um método utilizado para identificar, analisar e reportar padrões (aspectos comuns) na construção de temas (elaborados pelo pesquisador para capturar significados importantes e comuns em relação à questão de pesquisa) entre os dados (BRAUN e CLARKE, 2006). Riessman (2008) afirma que este tipo de análise preocupa-se essencialmente com o significado da narrativa, ou seja, com o que, como, a quem e por que os fatos foram ditos dentro do enredo, arremetendo assim ao universo de interesse social.

Em sua maioria os homens eram casados, católicos, residiam na cidade de Ribeirão Preto, eram aposentados, tinham casa própria, ensino fundamental 
incompleto e idade entre 61 a 81 anos. Todos realizaram prostatectomia radical e vivenciaram as sequelas da disfunção erétil e da incontinência urinária. Cinco deles ainda foram submetidos a sessões de radioterapia e três, concomitante ao tratamento médico, utilizaram terapias complementares com ervas.

Por meio da observação e registro em diário de campo apresentamos nossas interpretações aos fatos que ocorreram durante nosso acompanhamento com os participantes. Descrevemos nossas dificuldades e facilidades para compreender suas experiências como o fato de ser homem e investigar outros homens e frequentar alguns lugares típicos masculinos.

Depois de um longo período de reflexão das particularidades compartilhadas em campo e da busca por melhores evidências para interpretar estes sentidos, articulamos a apresentação dos fatos sob a forma de duas sinteses narrativas temáticas, denominadas de: enredos corporificados do câncer de próstata: normas, conflitos e descobertas; e masculinidades resignadas em tempos de crise: como nos vemos, enfrentamos e seguimos no nosso mundo social.

\section{Enredos corporificados do câncer de próstata: normas, conflitos e descobertas}

É apresentado as crenças, costumes e valores atribuídos ao corpo do homem na sociedade e em sua cultura as normas hegemônicas que regem a sua forma de lidar com o seu itinerário terapêutico, transformando o seu corpo um lócus de agência e objeto de sentidos durante o adoecimento.

A próstata foi reconhecida como um órgão presente no corpo masculino, mas por tratar-se de uma região regulada socialmente por normas e valores culturais, os homens identificaram a suas funções relacionadas a atributos biológicos e sociais como servir pra dar a pressão no jato da urina, na circulação do pênis, fornecer auxilio na hora do sexo, macheza (masculinidade), força, barba, pelo e músculo, sendo ela acima de tudo, imbricada da função de ligá-los as coisas do mundo e fazer com que as pessoas reconheçam que as coisas que eles fazem são diferentes das coisas das mulheres, ou seja, tem a função de firmar sua identidade de gênero, sua masculinidade.

Fazer os exames de toque e biópsia foi compreendido como se o ato tocasse no $\mathrm{H}$ masculino do homem, algo constrangedor e dolorido, comparado como 
uma prática de estupro, uma violação de sua identidade masculina, algo que os levavam a se sentirem derrotados, humilhados, torturados e que os deixavam envergonhados, pois colocava-os em situação subversiva da imagem masculina dominante que incorporavam quando estavam saudáveis. Como optaram pela busca do sistema de saúde médico para detecção da desordem de seus corpos, ao realizar os exames sentiam-se como um boi no matadouro..., diferentes de muitos outros homens agora, porque deixaram outro cara por a mão no seu corpo e literalmente tirar um pedaço (exame de biópsia). Como são regidos pela obrigação moral de afirmar por meio de seus atos que são masculinos, vivenciaram o dilema de viver entre a cruz e a espada, pois ou faziam o que acha que é certo ou faziam o que os outros acham correto, ou seja, ou cuidavam do seu corpo adoecido e deslocavam dos preceitos de sua masculinidade ou não cuidavam do corpo e mantinham sua moral.

resultado positivo para o CP foi interpretado pelos narradores como uma declaração de óbito, um sentimento de sofrimento e incerteza, por que segundo eles aquilo doeu mais que uma faca na barriga e não sabem o que farão da vida agora, por que em seu senso comum o câncer é uma doença maligna e amaldiçoada que quem pega está condenadoa perder a vida de um jeito ou de outro, pois é uma doença aguda que quem apresenta morre e nem sabe a placa do caminhão que atingiu. Assim o sentimento de surpresa foi comum entre os homens, que descreveram: parecia um bobão de queixo caído tentando procurar a explicação para isso.

No processo de busca por compreender a experiência do outro frente ao adoecimento, aprendemos com os narradores que seus modelos explicativos não são puros, certamente há um sincretismo influenciado pelo modelo médico, social e cultural que regem suas experiências. Dessa forma, concordamos com a perspectiva de Portugal (2016) ao defender que um dos sentidos das explicações da doença e seu itinerário terapêutico é estar atrelada aos fatores culturais, à rememoração de eventos e a experiências anteriores que são evocadas, reorganizadas e ressignificadas para sustentar à sua origem, a qual transcende o corpo individual e o diagnóstico na linguagem médica.

Com o auxilio do enfoque antropológico das masculinidades de Connell 
(2005), entendemos que cada cultura tem sua forma de realizar a leitura e defesa das masculinidades de seus membros, sendo a heteronormatividade um preceito defendido pela masculinidade hegemônica o qual apresenta domínio sob os homens considerados como subordinados a ordem de gênero.

Para a antropologia das masculinidades o corpo é compreendido como uma superficie sobre a qual a cultura imprime o seu gênero (CONNELL e PEARSE, 2015), assim articula-se como um substrato onde são incorporados os valores, normas e símbolos culturais arranjados em meio às estruturas de poder social que regulam os corpos em um padrão onde coabitam o biológico e o social em um permanente diálogo (SENKEVICS e POLIDORO, 2012).

\section{Masculinidades resignadas em tempos de crise: como nos vemos, enfrentamos e seguimos no nosso mundo social}

As transformações ocorridas sob o corpo social e biológico apresentados pelo homem por meio da perda da identidade masculina hegemônica e adoção de outras masculinas, onde o homem que era forte, potente e viril deu lugar para o homem "dependente, fraco e isolado", que lida com as adversidades da doença com seu agenciamento por meio da resignação do seu corpo e de suas práticas.

A experiência do homem com CP transita pelos estados de ser e sentir-se masculino dentro de sua cultura, fator este que envolve os significados sobre a condição de doente e que incidem diretamente sobre a sua identidade. Para Connell e Pearse (2015), o papel que o homem exerce na sociedade ocupa uma ordem de gênero que, em uma perspectiva hegemônica, é responsável pelo controle das relações e organizações dos sistemas locais e globais que regem a sociedade, pois aos papéis culturais intitulados aos homens residem preceitos de domínios, sexualidades, relações de poder e construção de hierarquias.

A identidade dos homens deste estudo, antes do adoecimento pelo CP, foi construída sobre a elucidação de vários elementos que traduzimos como físicos e comportamentais - tem que ter barba, falar grosso, fazer xixi em pé, gostar de mulher, ter ereção, força, tem que ter cabeça no lugar, corpo de homem, jeito de homem; relações entre os gêneros - homem é uma espécie de líder, é mais respeitado do que a mulher, não só em relação ao sexo mas em tudo e 
status social de poder - o homem é quem dá o suporte de moral para família, ele nasceu para ter domínio de tudo.

Corroborando com as narrativas incitadas pelos participantes, pesquisadores (CONNELL, 2005; SOUZA, 2010; VASCONCELOS, et al., 2016; MARTINS e MODENA, 2016) afirmam que a construção das masculinidades é um processo longo advindo desde o nascimento, onde os meninos são preparados culturalmente para responder às expectativas sociais referentes aos papéis que devem desempenhar, marcados por signos e símbolos de virilidade que concorrem para a formação de sua visão do que seja a masculinidade. Assim sendo, concordamos com o ponto de vista de Connell (2015), pois as masculinidades não se restringem às características biológicas e naturais, mas resultam de longos processos históricos e culturais, os quais são marcados por rupturas que interferem em suas relações, como a idade e o adoecimento.

Os narradores experimentaram transformações em suas masculinidades que foram desencadeadas pelo processo de envelhecimento. $O$ trecho a seguir extraído da narrativa ilustra esse aspecto e evidencia que a masculinidade do homem é ressignificada frente à doença e o envelhecimento: Quando chega a idade e a doença, as pessoas enxergam a gente de forma diferente e não nos veem tanto como homem, como acontece com quem é mais novo, então a gente se sente outra pessoa...

Connell (2005), Mesquita, Moreira e Maliski (2011) e Wenger (2013) aprofundam esta discussão descrevendo que o processo de adoecimento do corpo abala a manutenção da masculinidade hegemônica, pois apresenta dilemas na identidade, incluindo subordinação, passividade, reconhecimento de emoções e dependência. Especificamente no câncer, os homens reconfiguram sua identidade física e social e buscam preservar seus "status" de masculinidade familiar ao máximo possivel, todavia, a liminaridade é um processo inevitável, pois o desequilíbrio biológico reflete diretamente sobre as ações sociais alterando a identidade masculina.

O corpo masculino também foi modelado, construído e desconstruído pelo adoecimento e pela vida social, sendo os atos empregados na representação deste eu com o mundo, reflexo da experiência e do convivio com seu grupo. Neste 
cenário as masculinidades foram produzidas, e por este cenário apontamos que elas devem ser interpretadas. Portanto, para acompanhar nossa leitura sob a teia de sentidos que descortinamos dos nativos, faz-se necessário compreender que frente ao processo globalizante que permeia a cultura, como atestado por Connell (2015), as masculinidades nunca ocupam uma mesma posição dentro da cocha de retalhos, elas estão sempre alocando-se, modificando e ressignificando por meio das experiências da identidade do masculino.

\section{Um convite ao ponto de partida}

As narrativas focadas no acesso a experiência dos homens adoecidos pelo CP em seguimento ambulatorial, possibilitou descortinar uma realidade de um grupo local até então não explorado nos estudos antropológicos. Identificamos por meio de seus enredos as suas crenças, valores, símbolos e conhecimentos que dão sentido a maneira de lidar com seu adoecimento e sua masculinidade frente à cultura. Assim, defendemos que a cultura influenciou na experiência do homem adoecido pelo CP, transformando seu corpo biológico, social e sua masculinidade.

Na narrativa denominada "Enredos corporificados do câncer de próstata: normas, conflitos e descobertas" foi destacado as caracteristicas socioculturais e os modelos explicativos para o adoecimento do homem pelo $C P$, bem como o itinerário terapêutico adotado.A segunda narrativa, denominada de "Masculinidades resignadas em tempos de crise: como nos vemos, enfrentamos e seguimos no nosso mundo social", apresenta os sentidos atribuídos à experiência do adoecimento pelo CP após os tratamentos, na maneira como os homens lidam com as mazelas em seu corpo e as transformações que estas trazem em suas vidas sociais.

Compreendemos que a discussão em torno das masculinidades e saúde vão além das questões relacionadas a sexualidade. Elas se tecem na mesma teia de significados da vida e se rearranjam assim como a cultura em crenças, valores e simbolos que guiam os atos masculinos. Por este motivo, compreender os homens adoecidos, foi como mergulhar em um texto vivo onde pesquisador e pesquisado aprendem, compreendem e interpretam que o conceito de ser homem vai além de uma definição conotativa prejulgada pelo social, pois o homem "não cai do céu, ele brota do chão", e é criado desde a infância, e recriado no dia-a-dia ao 
longo do seu fluxo de vida por suas experiências.

Destacamos a importância dos profissionais de saúde apreenderem sobre a experiência do adoecido. Assim, convidamos a todos os leitores para apreciação desta obra em sua totalidade e apropiação dos sentidos e significados que instruiram os pesquisadores no caminho desta investigação.

\section{Referências}

BRASIL. Ministério da Saúde. Instituto Nacional de Câncer José Alencar Gomes da Silva. Coordenação Geral de Ações Estratégicas. Coordenação de Prevenção e Vigilância. Estimativa 2012: Incidência de Câncer no Brasil. Rio de Janeiro. Disponivel em: <http://www.inca.gov.br/estimativa/2012/>. Acesso em: 23 mai. 2012.

BRAUN, V.; CLARKE, V. Using thematic analysis in psychology. Qualitative Research in Psychology, v. 3, n. 2, 2006.

BURNS, S. M.; MAHALIK, J. Understanding how masculine gender scripts may contribute to men's adjustment following treatment for prostate cancer. American Journal of Men's Health, v.1, n. 4, 2007.

CECIL, R,; MCCAUGHAN, E.; PARAHOO, K. It's hard to take because I am a man's man': an ethnographic exploration of cancer and masculinity. European Journal of Cancer Care, v.19, n. 4, 2010.

CHAPPLE, A.; ZIEBLAND, S. Prostate cancer: embodied experience and perceptions of masculinity. Sociology of Health \& IIIness, v. 24, n. 6, 2002.

CONNELL, R. W. Masculinities. 2nd ed. Berkeley: University of California Press, 2005.

CONNELL, R. W.; MESSERSCHMIDT, J. W. Hegemonic masculinity: rethinking the concept. Gender \& Society, v. 19, n. 6, 2005.

CONNELL, R. PEARSE, R. Gender in World Perspective. 3nded.Polity, Cambridge. 2015.

CONNELL, R. Género e corporificação na sociedade mundial.Revista Lusófona de Estudos Culturais, Porto,v. 3, n. 1, 2015.

COURTENAY, W. Theorizing masculinity and men's health. In: Braom A; Tovey P. Men's health: body, identity and social context. United Kingdom: Wiley-Blackwell/John Wiley \& Sans Ltda Publication, 2010. Cap 1, p. 9-32.

CRANG, M.; COOK, I. Doing ethnographies. Los Angeles: Sage Publications, 2007. 
DAZIO, E. M. R.; SONOBE, H. M.; ZAGO, M. M. F. Os sentidos de ser homem com estoma intestinal por câncer colorretal: uma abordagem na antropologia das masculinidades. Revista Latino-Americana de Enfermagem, v. 17, n. 5, 2009.

EVANS, J. et al. Hegemonic masculinity: rethinking the concept. Journal of Men's Health, v. 8, n. 1, 2011.

EZER, $H$. et al. Psychosocial adjustment of men during the first year of prostate cancer. CancerNursing, v. 35, n. 2, 2012.

KAZER, M. W. et al. The experiences of unpartnered men with prostate cancer: a qualitative analysis. Journal of Cancer Survivorship, v. 5, n. 2, 2011.

KLEINMAN, A. The Illness Narratives: Suffering, Healing and the Human Condition. New York: Basic Books. 1988.

LANGDON, E. J. Cultura e processos de saúde e doença. In: JEOLÁS, L. S.; OLIVEIRA, M. (Orgs.). SEMINÁRIO SOBRE CULTURA, SAÚDE E DOENÇA, 1., 2003, Londrina. Anais... Londrina (PR): Ministério da Saúde; Universidade Estadual de Londrina e Secretaria Municipal de Ação Social/ Prefeitura Municipal de Londrina, jun. 2003. p. 91-107.

LOPES, M. H. B. M. et al. Life experiences of brasilian men with urinary incontinence and erectile dysfunction following radical prostatectomy. Wound, Ostomy and Continence Nurses Society, v. 39, n. 1, 2012.

MALISKI, S. L. et al. Renegotiating masculine identity after prostate cancer treatment. Qualitative Health Research, v. 18, n. 12, 2008.

MARTINS, A. M.; MODENA, C. M. Estereótipos de gênero na assistência ao homem com câncer: desafios para a integralidade. Trabalho, Educação e Saúde, Rio de Janeiro, v. 14, n. 2, 2016.

MESQUITA, M. G.; MOREIRA, M. C.; MALISKI, S. L. "But I'm (became) different": cancer generates reprioritizations in masculine identity. Cancer Nursing. v. 34, n. 2, 2011.

MIGOWSKI, A.; SILVA, G. A. Sobrevida e fatores prognósticos de pacientes com câncer de próstata clinicamente localizado. Revista de Saúde Pública, v. 44, n. 2, 2010.

NATIONAL INSTITUTE OF HEALTH. NATIONAL CANCER INSTITUTE. General information about prostate cancer: Health Professional Version, 2012. Disponivel em http://www.cancer.gov/cancertopics/pdq/treatment/ prostate/HealthProfessional. Acessoem: 23 maio 2012.

OLLIFE, J.; THORNE, S. Men, masculinities, and prostate câncer: Australian and Canadian patient perspectives of communication with male physicians. Qualitative Health Research, v. 17, n. 2, 2007. 
POPE, C.; MAYS, N. Pesquisa qualitativa na atenção à saúde. Tradução de Ananyr Porto Fajardo. 3th ed. Porto Alegre: Artmed, 2009.

PORTUGAL, C. M. Entre o consultório e o terreiro: mediações, ruídos e silenciamentosnositineráriosterapêuticos de adeptos do candomblé. Revista Eletrônica de Comunicação, Informação \& Inovação em Saúde, Rio de Janeiro, v. 10, n. 1, p.1-14, jan. 2016.

RIESSMAN, C. K. Narrative methods for the human sciences. Los Angeles: Sage Publications, 2008.

RHODEN, E. L.; AVERBECK, M. A. Câncer de próstata localizado. Revista da AMRIGS, v. 54, n. 1, 2010.

SENKEVICS, A. S.; POLIDORO, J. Z. Corpo, gênero e ciência: na interface entre biologia e sociedade. Revista da Biologia v.9 n. 1, 2012.

SOUZA, E. Olhares sobre a identidade masculina. Protestantismo em Revista, São Leopoldo, v. 21, 2010.

VASCONCELOS, A. C. S.; MONTEIRO, R. J. S.; FACUNDES, VERA, L. D.; TRAJANO, M. F. C.; GONTIJO, D. T. Eu virei homem!: a construção das masculinidades para adolescentes participantes de um projeto de promoção de saúde sexual e reprodutiva. Saúde e sociedade, São Paulo, v. 25, n. 1, 2016.

WALL, D.; KRISTJANSON, L. Men, culture and hegemonic masculinity: understanding the experience of prostate cancer. Nursing Inquiry, v. 12, n.2, 2004.

WENGER, L. M. Living under assault: men making sense of cancer. European Journal of Cancer Care, London, v.22, n 3, 2013.

Recebido em dezembro de 2017

Aceito para publicação em junho de 2018 